\title{
Quantitative Sensory Testing
}

National Cancer Institute

\section{Source}

National Cancer Institute. Quantitative Sensory Testing. NCI Thesaurus. Code C155860.

A non-invasive method for diagnosing peripheral nervous system disorders. It determines an individial's sensation and pain thresholds for cold and warm temperatures, and vibration sensation threshold by stimulating the skin and comparing the results to standard values. 